\title{
Critical behavior of roughening transitions in parity-conserving growth processes
}

\author{
Haye Hinrichsen ${ }^{1}$ and Géza Ódor ${ }^{2}$ \\ ${ }^{1}$ Max-Planck-Institut für Physik komplexer Systeme, Nöthnitzer Straße 38, D-01187 Dresden, Germany \\ ${ }^{2}$ Research Institute for Technical Physics and Materials Science, P. O. Box 49, H-1525 Budapest, Hungary
}

(July 14, 1999)

\begin{abstract}
We investigate a class of parity-conserving solid-on-solid models which describe the growth of an interface by the deposition and evaporation of dimers. As a key feature of the models, evaporation of dimers takes place only at the edges of terraces, leading to a roughening transition between a smooth and a rough phase. We consider several variants of growth models in order to identify universal and nonuniversal properties. Moreover, a parity-conserving polynuclear growth model is proposed. All variants display the same type of universal critical behavior at the roughening transition. Because of parity-conservation, the critical behavior at the first few layers can be explained in terms of unidirectionally coupled branching annihilating random walks with even number of offspring.
\end{abstract}

PACS numbers: 64.60.Ak, 05.40.+j, 82.20.-w.

\section{INTRODUCTION}

In the present work we continue the investigation of a certain class of nonequilibrium models for interfacial growth by adsorption and desorption [1]. The models may be used to describe a layer-by-layer growth process of a $d$-dimensional surface by deposition and evaporation of dimers which are aligned with the surface. Upon adsorption the dimers dissociate into two atoms at neighboring lattice sites. It is assumed that the atoms cannot diffuse on the surface. Furthermore, atoms cannot evaporate from the interior of completed layers. Only pairs of neighboring atoms at the edges of terraces are able to form a dimer and evaporate back into the gas phase.

The most interesting property of this class of growth processes is the emergence of a roughening transition from a smooth to a rough phase at a certain critical ratio of the adsorption and desorption rates [2]. In contrast to equilibrium growth models and nonequilibrium growth processes described by the Kardar-Parisi-Zhang (KPZ) equation [3], where roughening transitions take place only in $d \geq 2$ dimensions, the models discussed in the present work exhibit a robust roughening transition even in one spatial dimension. As will be shown below, the scaling properties of the interface at the transition differ significantly from the usual scaling laws for roughening interfaces, i.e., the models exhibit anomalous roughening properties.

The motivation to consider growth processes of dimers originates in recent studies of similar interface models for adsorption and desorption of monomers [4. In these models individual atoms are adsorbed with probability $p$ on each lattice site, whereas atoms evaporate with probability $1-p$ solely at the edges of terraces. It was shown that monomer models of this kind exhibit a roughening transition which is closely related to the universality class of directed percolation (DP) [5]. A more detailed analysis revealed that the critical behavior of the first few layers can be explained in terms of unidirectionally coupled DP processes [6]. It turned out that such hierarchies of cou- pled DP processes not only describe the monomer models of Ref. [4] but also various other DP-related growth processes, including polynuclear growth (PNG) models [7], certain models for fungal growth [8], and a recently introduced model designed for real-space renormalization [9]. Thus, the concept of 'coupled DP' characterizes a whole universality class of roughening transitions [10].

DP itself is the generic universality class for phase transitions into trapped (absorbing) states and is known to be extremely robust with respect to the choice of the dynamic rules. The DP conjecture [1] states that in a random process with short-range interactions any transition from a fluctuating active phase into a single absorbing state should belong to the DP class, provided that the dynamics is characterized by a single-component order parameter without additional symmetries. NonDP critical behavior is expected in systems where one of these requirements is violated. An important example is the so-called parity-conserving (PC) universality class for phase transitions into absorbing states in which the parity of the particle number is conserved. In one dimension, this conservation law can also be interpreted as a $Z_{2}$-symmetry between two different absorbing states. The PC class is represented most prominently by branching annihilating random walks with two offspring (BAW2) 12 14. Other examples include nonequilibrium kinetic Ising models [15], interacting monomerdimer models [16], as well as models with two symmetric absorbing states [17. It is therefore near at hand to investigate the question how the physical properties change if the DP mechanism of the growth models in Ref. [4] is replaced by a parity-conserving dynamics. To this end we modify the dynamic rules of these models by using dimers instead of monomers which adsorb with probability $p$ and desorb at the edges of terraces (including solitary dimers) with probability $1-p$. As dimers consist of two atoms, the number of particles at each height level is conserved modulo 2. As shown in Ref. [1], the model exhibits a robust roughening transition which is related to the $\mathrm{PC}$ class. 

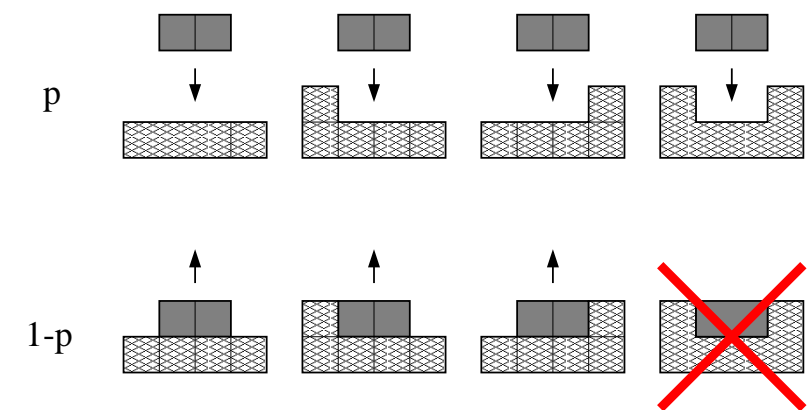

FIG. 1. Variant $\mathrm{A}$ in $d=1$ dimension: Dimers are adsorbed with probability $p$ and desorbed at the edges of terraces with probability $1-p$. Evaporation from the middle of plateaus is not allowed.

In the following we present a detailed numerical analysis of the critical behavior of parity-conserving growth processes. One of our aims is to clearly identify universal and nonuniversal properties. To this end we consider four variants of the model, a restricted version with random sequential updates, an unrestricted version as well as the corresponding variants with parallel updates. It turns out that all variants exhibit the same type of critical behavior at the roughening transition. In particular, the critical behavior at low-lying levels can successfully be described in terms of unidirectionally coupled PC processes, generalizing the concept of 'coupled DP'. Above the transition, however, restricted and unrestricted variants display different properties.

The paper is organized as follows. In Sect. II we define four variants of the dimer growth model. Their phenomenological properties are described in Sect. III. Sects. IV and $\mathrm{V}$ investigate the critical properties of the interface width and the densities of exposed sites at the first few layers. We also discuss aspects of spontaneous symmetry breaking and unusual scaling properties for random initial conditions. The critical behavior at the roughening transition can partly be explained in terms of unidirectionally coupled PC processes for which we propose a field-theoretic formulation in Sect. VI. As will be shown in Sect. VII, it is even possible to construct a polynuclear growth model which belongs to the same universality class. Our conclusions are summarized in Sect. VIII.

\section{MODELS DEFINITION}

The class of models may be introduced in terms of a $d$-dimensional interface which evolves by adsorption and desorption of dimers. As a key feature desorption may only take place at the edges of a plateau, i.e. at sites which have at least one neighbor at a lower height. The dimer growth model is defined on a $d$-dimensional square lattice with $L^{d}$ sites and periodic boundary conditions. Each site $i$ is associated with an integer height variable

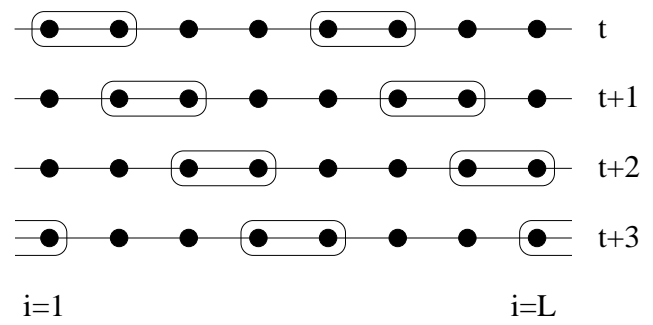

FIG. 2. Variants C and D: Cyclic parallel updates on four different sublattices in a one spatial dimension.

$h_{i} \in \mathbb{Z}$. We consider four variants $\mathrm{A}, \mathrm{B}, \mathrm{C}, \mathrm{D}$ of the model which differ by their dynamic rules.

Variant A is a restricted solid-on-solid (RSOS) model evolving by random sequential updates. For each attempted update a pair of adjacent sites $i$ and $j$ is selected at random. If the heights $h_{i}$ and $h_{j}$ are equal a dimer is adsorbed with probability $p$

$$
\begin{aligned}
& h_{i} \rightarrow h_{i}+1 \\
& h_{j} \rightarrow h_{j}+1
\end{aligned}
$$

or desorbed with probability $1-p$

$$
\begin{aligned}
& h_{i} \rightarrow h_{i}-1 \\
& h_{j} \rightarrow h_{j}-1
\end{aligned} \quad \text { if } \min _{k \in<i, j>} h_{k}<h_{i}
$$

where $k$ runs over the nearest neighbors of sites $i$ and $j$. An attempted update is rejected if it violated the RSOS constraint

$$
\left|h_{i}-h_{j}\right| \leq 1
$$

i.e., the heights at neighboring sites may differ by at most one step. A Monte-Carlo sweep consisting of $L^{d}$ local update attempts corresponds to a time increment $t \rightarrow$ $t+1$. For $d=1$ the dynamic rules are shown in Fig. 1. Notice that the rules are translationally invariant in time, space as well as in height direction. Thus, the layer at $h=0$ has no particular physical meaning, although we will often use a flat interface at zero height as initial condition.

Variant B is an unrestricted solid-on-solid (SOS) model which is defined by the same rules without restriction (3). Although unrestricted growth is less realistic, it exhibits essentially the same type of critical behavior at the roughening transition, supporting the claim of universality.

Variants C and D are counterparts of A and B employing parallel updates. More precisely, their lattice is divided into several sublattices in a way that synchronous updates on each sublattice according to the rules (1) and (2) do not overlap. In $d=1$ dimension at least three sublattices are needed. However, for technical reasons it is more convenient to work with four different sublattices, as illustrated in Fig. 2. We implement this update scheme on a parallel computer with 24000 string 


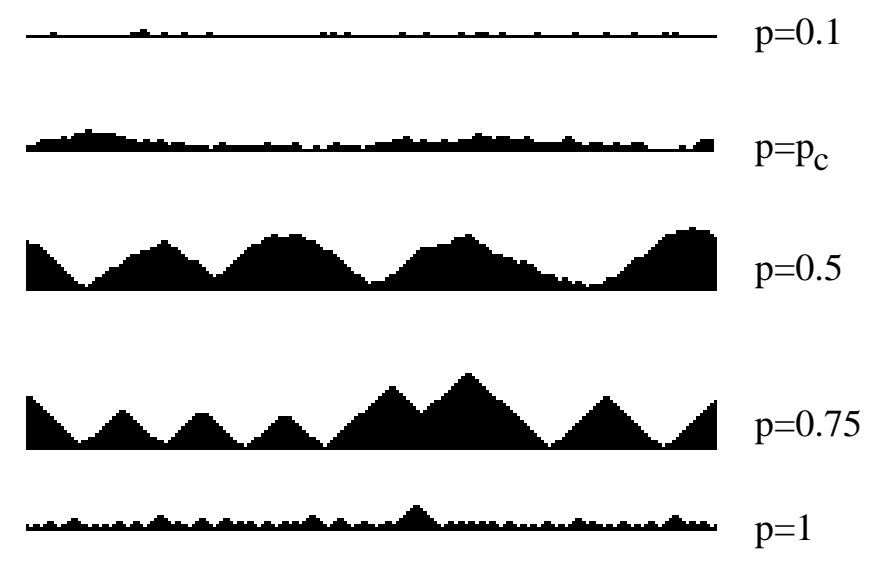

FIG. 3. Typical interface configurations of the restricted dimer model (variant A) for various values of $p$ (see text).

processors. Associating an individual processor with each lattice site we are able to perform efficient simulations. Further details on massive parallel computing are given in Ref. 18].

\section{PHENOMENOLOGY OF THE INTERFACE DYNAMICS}

Although the dimer models are defined in arbitrary spatial dimensions, this work is restricted to the onedimensional case $d=1$. Clearly, the morphology of the interface depends on the growth rate $p$. Let us first consider the restricted variants (see Fig. 3). If $p$ is very small, only a few dimers are adsorbed at the surface, staying there for a short time before they evaporate back into the gas phase. Thus, the interface is anchored to the actual bottom layer and does not propagate. As $p$ increases, a growing number of dimers covers the surface and large islands of several layers stacked on top of each other are formed. Approaching a certain critical threshold $p_{c}$ the mean size of the islands diverges and the interface evolves into a rough state.

Above $p_{c}$ one may expect the interface to detach from the bottom layer in the same way as the interface of monomer models starts to propagate in the supercritical phase. However, since dimers are adsorbed at neighboring lattice sites, solitary unoccupied sites may emerge. These pinning centers prevent the interface from moving and lead to the formation of 'droplets' (see Fig. 3). Due to interface fluctuations, the pinning centers can slowly diffuse to the left and to the right. When two of them meet at the same place, they annihilate and a larger droplet is formed. Thus, although the interface remains pinned, its roughness increases continuously.

As realized in 19], a second transition takes place at $p=0.5$ where the width grows most rapidly. In this case the droplets reach an almost triangular shape with unit slope, i.e., the surface becomes faceted. The tilted
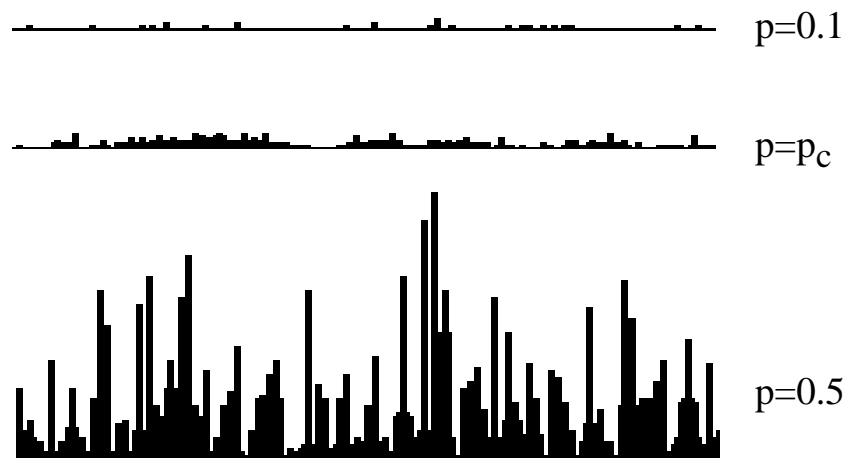

FIG. 4. Typical interface configurations of the unrestricted dimer model (variant B) for various values of $p$. At the transition $p=0.5$ large spikes of stacked dimers are formed. For $p>0.5$ these spikes are biased to grow with constant velocity.

surface of the droplets fluctuates predominantly by randomly moving triplets of sites at equal height along 'staircases' of unit slope. Inspecting the dynamic rules it is easy to verify that these 'landings' move upwards with probability $p$ and downwards with probability $1-p$. This explains why the faceting transition takes place exactly at $p=0.5$. For $p>0.5$ the fluctuations are confined to an exponentially small region at the top of the droplets. Therefore, the faceted interface coarsens on a logarithmic time scale. A pathological situation emerges for $p=1$ where evaporation of dimers is forbidden. As the pinning centers, once formed, cannot diffuse the interface quickly evolves into a frozen configuration.

To summarize, the restricted variants $\mathrm{A}$ and $\mathrm{C}$ display three different phases, a smooth phase $p<p_{c}$, a rough phase $p_{c}<p<0.5$, and a faceted phase $p>0.5$. The phase structure of the unrestricted variants $B$ and $D$ is very similar (see Fig. (4). They too exhibit a roughening transition at a certain critical threshold $p_{c}$. However, the rough phase and the transition at $p=0.5$ are different in character due to the formation of spikes, as will be described below.

\section{CRITICAL BEHAVIOR OF THE INTERFACE WIDTH}

The morphology of a growing interface is usually characterized by its width

$$
W(L, t)=\left[\frac{1}{L} \sum_{i} h_{i}^{2}(t)-\left(\frac{1}{L} \sum_{i} h_{i}(t)\right)^{2}\right]^{1 / 2} .
$$

In order to investigate the scaling properties of the width, we perform Monte-Carlo simulations starting from a flat interface $h_{i}(0)=0$ and get the following results:

$a$. The smooth phase $p<p_{c}$ : Fig. 5 shows the temporal evolution of $W^{2}(t)$ for different values of $p \leq p_{c}$ 

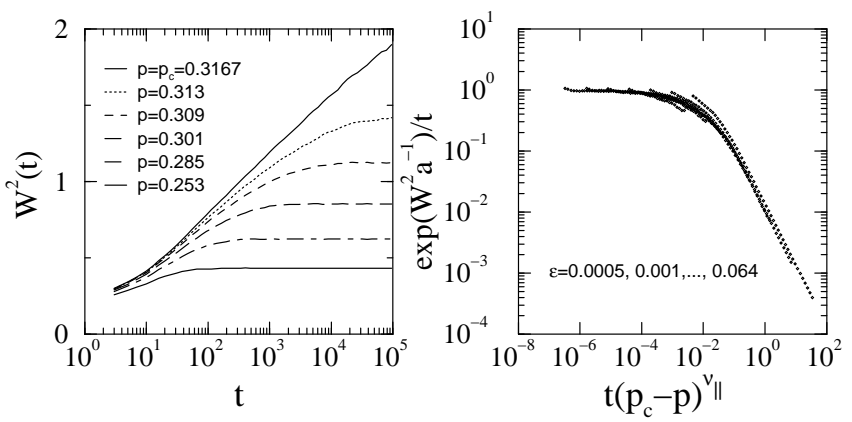

FIG. 5. Left: The squared width $W^{2}(t)$ measured in variant $\mathrm{A}$ for various values of $p \leq p_{c}$. Right: Data collapse according to Eq. (5), visualizing the scaling function $F$. Throughout the whole paper $t$ is measured in units of Monte-Carlo sweeps.

measured in model A with $L=10000$ sites. As can be seen, the width first increases as $W(t) \sim \sqrt{\ln t}$ until it saturates at some constant value. The initial logarithmic increase suggests the scaling form

$$
W^{2}(t, \epsilon) \simeq a \ln \left[t F\left(t \epsilon^{\nu \|}\right)\right]
$$

where $a$ is an amplitude factor and $\epsilon=\left|p-p_{c}\right|$ denotes the distance from criticality. The exponent $\nu_{\|}$describes the singular behavior of the temporal correlation length $\xi_{\|} \sim \epsilon^{-\nu_{\|}}$close to the roughening transition. $F$ is a universal scaling function with the asymptotic behavior

$$
F(\zeta)= \begin{cases}\text { const } & \text { if } \zeta \rightarrow 0 \\ \zeta^{-1} & \text { if } \zeta \rightarrow \infty\end{cases}
$$

In order to estimate $a$ and $\nu_{\|}$we adjust these quantities in a way that the curves for $\exp \left(W^{2} / a\right) / t$ versus $t \epsilon^{\nu_{\|}}$ collapse onto a single one. Using the estimates $p_{c}=$ $0.317(1), \nu_{\|}=2.4(4)$, and $a=0.17(1)$ we obtain a fairly convincing data collapse (see right hand graph of Fig. 同). However, we observe considerable deviations for larger values of $\epsilon$. These deviations may indicate corrections of the scaling form (5) in the off-critical regime and will be analyzed in Sect. VE. Similar results are obtained for variants $\mathrm{B}, \mathrm{C}$, and $\mathrm{D}$.

$b$. The roughening transition at $p=p_{c}$ : At the roughening transition the interface width of an infinite system grows logarithmically as $W \sim \sqrt{\ln t}$. A similar logarithmic behavior has also been observed at the roughening transition of monomer models [4]. The logarithmic time dependence suggests the finite-size scaling form 20]

$$
W^{2}(L, t) \simeq a \ln \left[t G\left(t / L^{z}\right)\right]
$$

where $z$ denotes the dynamic exponent. $G$ is a universal scaling function with the same asymptotic behavior as in Eq. (6). Thus, the interface of finite systems is
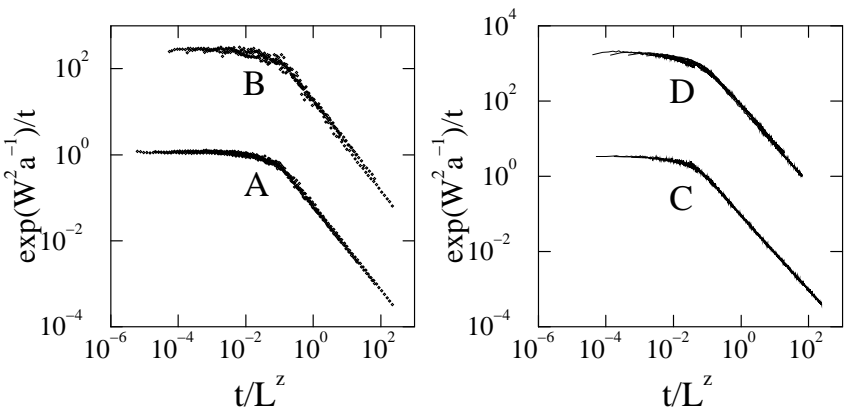

FIG. 6. Finite-size scaling of the interface width $W(L, t)$ for variants A-D. The graphs show data collapses according to the scaling form $($ (7) , visualizing the scaling function $G$.

expected to saturate at a constant value $W(L) \sim \sqrt{\ln L}$. Our numerical results are summarized in Fig. 6. Plotting $\exp \left(W^{2} / a\right) / t$ against $t / L^{z}$ in the time interval $10 \leq t \leq$ $10^{5}$ for system sizes $L=32,64, \ldots, 4096$, we determine $a$ and $z$ by data collapse. For all variants we obtain accurate data collapses. The estimates for $z$ coincide in all cases, indicating universal properties of the roughening transition independent of the RSOS constraint and the type of updates (see Table $\mathbb{1}$ ). In fact, as we will argue in the following Section, $z \simeq 1.75$ is the dynamic exponent of the PC universality class. The amplitude $a$, however, is nonuniversal. A very accurate data collapse is obtained by simulations of variant $\mathrm{C}$ on a parallel computer and highly supports the validity of the scaling form (7). It would be interesting to confirm this scaling form by direct diagonalization of the transfer matrix [21].

$c$. The rough phase $p_{c}<p<0.5$ : In the rough phase the formation of pinning centers prevents the interface from propagating. By diffusion and pairwise annihilation of the pinning centers the width increases very slowly. Since the width displays a rather inconclusive scaling behavior, it was impossible conjecture an appropriate scaling form [22]. It seems that the width initially increases algebraically until it slowly crosses over to a logarithmic increase $W(t) \sim \sqrt{a \ln t}$. The amplitude $a$ and the crossover time grow with $p$ and diverge at the transition at $p=0.5$. The crossover time provides a typical time scale of the dynamics in the rough phase. Apart from that the roughening transition is associated with another temporal correlation length $\xi_{\|}=\left(p-p_{c}\right)^{\nu_{\|}}$. Thus, the rough phase is characterized by a complicated interplay of at least two different time scales.

$d$. The transition at $p=0.5:$ The restricted as well as the unrestricted variants undergo a second phase transition at $p=0.5$ where the width increases algebraically with time as $W \sim t^{\tilde{\beta}}$. Let us first consider the restricted case where the transition can be interpreted as a faceting transition between a rough and a faceted phase 19]. As the width increases algebraically, we expect ordinary Family-Vicsek scaling [23] of the form

$$
W(L, t) \sim L^{\tilde{\alpha}} f\left(t / L^{\tilde{\alpha} / \tilde{\beta}}\right),
$$




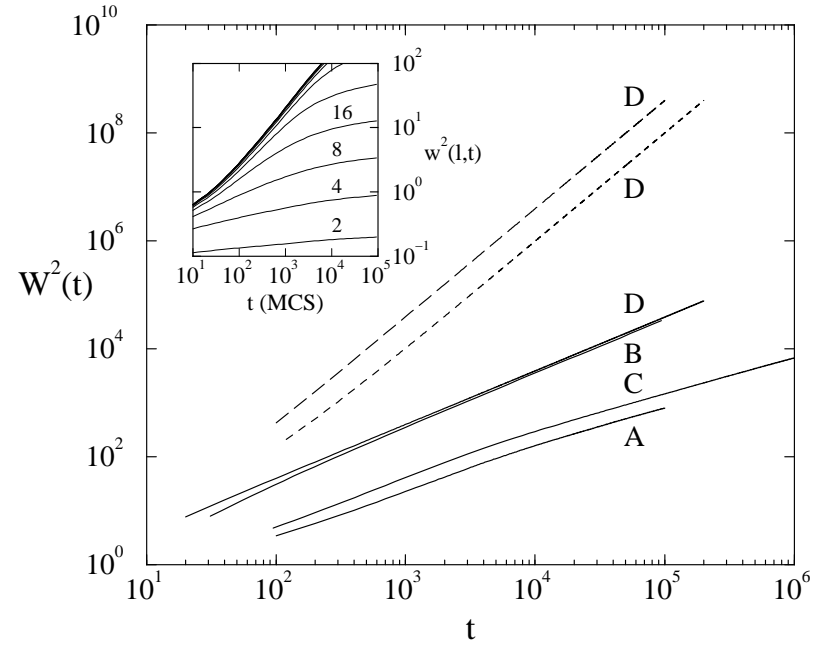

FIG. 7. Power-law roughening of the width in large systems. The figure shows the squared width as a function of time at the transition $p=0.5$ (solid lines), as well as above the transition for $p=0.6$ (dashed line) and $p=0.7$ (long-dashed line). The inset shows the squared local width of variant $A$ for increasing box sizes $l=2,4,8,16, \ldots$ as a function of time (see text).

where $\tilde{\alpha}$ and $\tilde{\beta}$ are the roughening and the growth exponents, respectively. However, finite size simulations (not shown here) reveal a more complex behavior. After an initial short-time regime we observe a long transient extending over two decades in time where the interface roughens with the exponents $\tilde{\alpha} \simeq 0.5$ and $\tilde{\beta} \simeq 0.45$. After approximately 5000 time steps both variants $\mathrm{A}$ and $\mathrm{C}$ cross over to a different regime with $\tilde{\beta} \simeq 0.33$. By a finite size data collapse we find a roughening exponent $\tilde{\alpha} \simeq 1.2$ and a large dynamic exponent $\tilde{z}=\tilde{\alpha} / \tilde{\beta} \simeq 3$. These results are consistent with the findings of Ref. 19. In Fig. 7 the crossover appears as a slight change of the slope, suggesting the faceting transition at $p=0.5$ not to be fully scale-invariant but characterized by a finite time scale. Moreover, as shown in the inset of Fig. 7, the faceting transition displays anomalous scaling properties [24], i.e., the local width $w(l, t)$ measured in boxes of sizes $l=2,4,8,16, \ldots$ does not saturate after a short time. Even for small box sizes it continues to increase over several time decades until it saturates due to the RSOS constraint.

Let us now consider the unrestricted variants B and D. As can be seen in Fig. 田, their phase transition at $p=0.5$ is different in character. Since large spikes are formed, the surface roughens much faster with a growth exponent of $\tilde{\beta} \simeq 0.5$ (see Fig. 17). In fact, the interface evolves into configurations with large columns of dimers separated by pinning centers. These spikes can grow or shrink almost independently. Thus, the interface roughens by a purely diffusive mechanism as $W(t) \sim \sqrt{t}$. As the columns are spatially decoupled, the width does not saturate in finite systems, i.e., the dynamic exponents $\tilde{\alpha}$ and $\tilde{z}$ have no

\begin{tabular}{||c||c|c|c|c||}
\hline \hline variant & $\mathrm{A}$ & $\mathrm{B}$ & $\mathrm{C}$ & $\mathrm{D}$ \\
\hline restriction & yes & no & yes & no \\
updates & random & random & parallel & parallel \\
\hline$p_{c}$ & $0.3167(2)$ & $0.292(1)$ & $0.3407(1)$ & $0.302(1)$ \\
$a$ & $0.172(5)$ & $0.23(1)$ & $0.162(4)$ & $0.19(1)$ \\
$z$ & $1.75(5)$ & $1.75(5)$ & $1.74(3)$ & $1.77(5)$ \\
$\delta_{0}$ & $0.28(2)$ & $0.29(2)$ & $0.275(10)$ & $0.29(2)$ \\
$\delta_{1}$ & $0.22(2)$ & $0.21(2)$ & $0.205(15)$ & $0.21(2)$ \\
$\delta_{2}$ & $0.14(2)$ & $0.14(3)$ & $0.13(2)$ & $0.14(2)$ \\
$\theta / \nu_{\|}$ & $0.765(10)$ & $0.765(10)$ & $0.753(3)$ & no result \\
\hline$\tilde{\alpha}$ & $1.2(1)$ & undefined & $1.25(5)$ & undefined \\
$\tilde{\beta}$ & $0.34(1)$ & $0.50(1)$ & $0.330(5)$ & $0.49(1)$ \\
\hline \hline
\end{tabular}

TABLE I. Numerical estimates for the four variants of the dimer model at the roughening transition $p=p_{c}$ (upper part) and the transition $p=0.5$ (lower part).

physical meaning.

$e$. The faceted/free phase: For $p>0.5$ the restricted models $\mathrm{A}$ and $\mathrm{C}$ evolve into faceted configurations (see Fig. 3). As shown in Ref. [19], the width first increases algebraically until the pinning centers become relevant and the system crosses over to a logarithmic increase of the width. Therefore, the faceted phase may be considered as a rough phase. The unrestricted models $\mathrm{B}$ and $\mathrm{D}$, however, evolve into spiky interface configurations. The spikes are separated and grow independently by deposition of dimers. Therefore, the interface width increases linearly with time, defining the free phase of the unrestricted models.

\section{CRITICAL PROPERTIES OF THE FIRST FEW LAYERS}

\section{A. Relation to the PC class}

In this Section we investigate the roughening transition at $p=p_{c}$ in more detail. In order to understand its relation to the PC class let us consider all sites at the bottom layer $h_{i}=0$ as $A$-particles. Adsorption and desorption processes correspond to certain effective reactions of the $A$-particles. For example, the adsorption of a dimer at the bottom layer corresponds to a pair-annihilation process $2 A \rightarrow \varnothing$ at rate $p$. Similarly, when a dimer evaporates, two $A$-particles are created. However, since dimers can only evaporate at the edges of terraces, this process always requires the presence of another neighboring $A$ particle, giving rise to an effective reaction $A \rightarrow 3 A$ at rate $1-p$. These two processes compete one another and resemble a branching-annihilating random walk with two offspring (BAW2) [12 13] which is known to belong to the $\mathrm{PC}$ universality class. In one spatial dimension the PC class is characterized by three critical exponents 


$$
\beta=0.92(2), \quad \nu_{\|}=3.22(6), \quad \nu_{\perp}=1.83(3) .
$$

The two scaling exponents $\nu_{\|}$and $\nu_{\perp}$ are associated with temporal and spatial correlation lengths

$$
\xi_{\|} \sim\left|p_{c}-p\right|^{-\nu_{\|}}, \quad \xi_{\perp} \sim\left|p_{c}-p\right|^{-\nu_{\perp}}
$$

which diverge at the transition. The order parameter of the PC transition is the density of $A$-particles, corresponding to the density $n_{0}$ of exposed sites at the bottom layer. Thus, in the smooth phase $n_{0}$ should scale as

$$
n_{0} \sim\left(p_{c}-p\right)^{\beta_{0}}
$$

where $\beta_{0}=\beta$ is the density exponent of the PC class. More generally, we expect $n_{0}$ to obey the scaling form

$$
n_{0}(t, L, \epsilon) \sim t^{-\beta_{0} / \nu_{\|}} \Phi_{0}\left(t \epsilon^{\nu_{\|}}, t / L^{z}\right),
$$

where $\Phi_{0}$ is a universal scaling function. As will be shown below, numerical simulations confirm the validity of this scaling form. This may be surprising since the $A$-particles do not evolve independently, but are coupled to dynamic processes at higher levels of the interface. However, in the present models this feedback from higher levels is comparatively weak and does not seem to affect the critical behavior of the $A$-particles at the roughening transition.

In order to investigate the critical behavior at higher levels, let us introduce the densities of sites $i$ with $h_{i} \leq k$

$$
n_{k}=\frac{1}{L} \sum_{j=0}^{k} \sum_{i} \delta_{h_{i}, j} . \quad k=1,2, \ldots
$$

Following the ideas of Ref. [A] we expect $n_{1}, n_{2}, \ldots$ to scale in the same way as $n_{0}$ with certain critical exponents $\beta_{k}, \nu_{\perp, k}$, and $\nu_{\|, k}$. However, since the correlation lengths of neighboring layers should coincide, the exponents $\nu_{\perp, k}$ and $\nu_{\|, k}$ should not depend on $k$, leading to the scaling form

$$
n_{k}(t, L, \epsilon) \sim t^{-\beta_{k} / \nu_{\|}} \Phi_{k}\left(t \epsilon^{\nu_{\|}}, t / L^{z}\right) .
$$

Our numerical results support the validity of this scaling form for $k \geq 1$ in an intermediate scaling regime.

\section{B. Estimation of $p_{c}$ and $\delta_{k}$}

In order to determine the critical exponents accurately, we perform time-dependent simulations of variant $\mathrm{C}$ on a parallel computer. Using a large lattice of 24000 sites (associating one processor with each lattice site) we measure $n_{0}, \ldots, n_{2}$ as functions of time up to $2 \cdot 10^{5}$ time steps averaged over $10^{4}$ samples. At criticality, the densities are expected to decay as
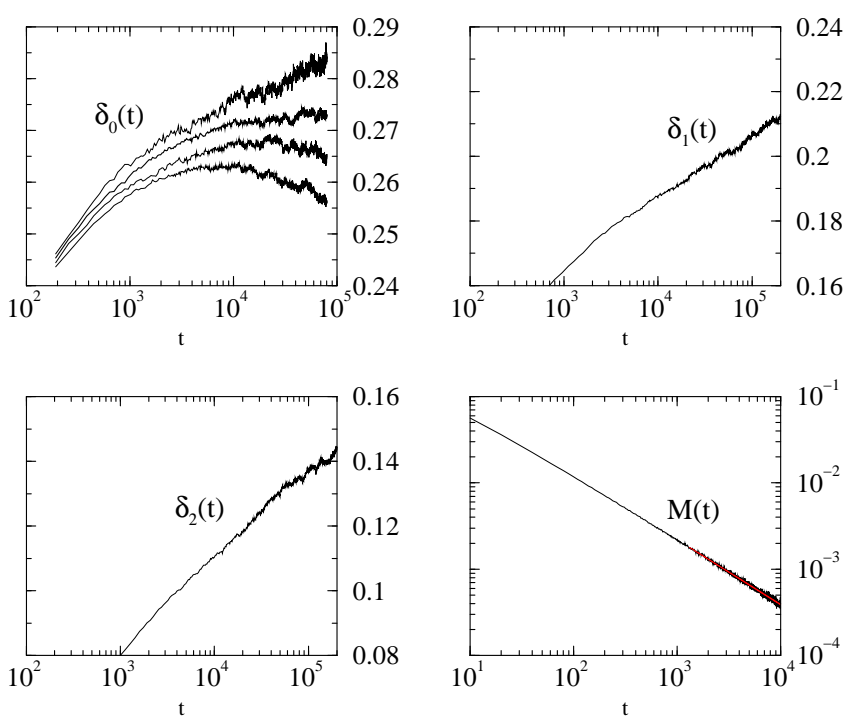

FIG. 8. Effective exponents $\delta_{k}(t)$ measured in model $\mathrm{C}$ with 24000 sites averaged over $10^{4}$ samples. The first panel shows $\delta_{0}(t)$ with $p$ ranging from 0.3406 to 0.3409 from top to bottom, leading to the estimates $p_{c}=0.3407(1)$ and $\delta_{0}=0.275(10)$. The curves for $\delta_{1}(t)$ and $\delta_{2}(t)$ at $p=p_{c}$ are shown in panel 2 and 3 . The last panel shows the magnetization $M$ as a function of time.

$$
n_{k}(t) \sim t^{-\delta_{k}}
$$

where $\delta_{k}=\beta_{k} / \nu_{\|}$. In Fig. 8 the effective exponent $\delta_{0}(t)$ (i.e., the local slope of $n_{0}(t)$ in a log-log plot, see [25]) is plotted against $t$ in a logarithmic scale for various values of $p$. As can be seen, the curve that appears to become approximately horizontal for large $t$ corresponds to the critical point $p_{c}=0.3407(1)$. For the exponent $\delta_{0}$ we obtain the estimate

$$
\delta_{0}=0.275(10)
$$

which is in agreement with the expected value of the $\mathrm{PC}$ class $\delta=\beta / \nu_{\|}=0.285(5)$.

The estimation of the exponents $\delta_{k}$ at higher levels is more difficult. Averaging $\delta_{k}(t)$ over two time decades we obtain the estimates

$$
\delta_{1}=0.205(15), \quad \delta_{2}=0.13(2) .
$$

Less accurate but compatible results for the other variants are listed in Table ש. Thus, the critical behavior at the first few layers is universal and does not depend on the RSOS constraint and the type of updates. However, as can be seen in Fig. 8, the effective exponents $\delta_{1}(t)$ and $\delta_{2}(t)$ do not saturate at a constant value in the long-time limit. Instead they continue to increase on a logarithmic time scale. At present it is not clear whether the effective exponents will eventually saturate after very long time. The drift of the exponents may indicate violations of scaling in the long-time limit which are neither related 
to finite-size effects nor to numerical errors in the value of $p_{c}$. Thus, the estimates (17) have to be taken with care. Similar violations of scaling have been observed in the monomer case and seem to be an intrinsic property of roughening transitions driven by absorbing-state transitions. We will come back to this problem in Sect. VI.

\section{Spontaneous symmetry breaking}

The growth processes considered in this paper are translationally invariant in height direction. This symmetry is spontaneously broken in the smooth phase where the models select one of the heights as the bottom layer of the interface. In order to quantify this symmetry breaking we consider an order parameter

$$
M=\frac{1}{L} \sum_{j}(-1)^{h_{j}},
$$

where $j$ runs over all lattice sites. This order parameter can take positive or negative values and thus reminds of a magnetization. In the stationary state of the smooth phase $|M|$ is positive and vanishes at the roughening transition. As in the monomer case, we expect the magnetization to scale near the roughening transition as

$$
|M| \sim \epsilon^{\theta}
$$

where $\theta$ is a critical exponent. Thus, starting from a flat interface, $|M|$ should decrease at criticality as $t^{-\theta / \nu_{\|}}$. Performing numerical simulations (see Fig. 8 and Table II) we find $\theta / \nu_{\|} \simeq 0.76$ for both the restricted and the unrestricted variants, suggesting that $\theta$ is a universal critical exponent. As will be shown in the following Section, this exponent can also be seen in unidirectionally coupled $\mathrm{PC}$ processes. Its numerical value $\theta \simeq 2.5$ is much larger than in the monomer case where the value was found to be 0.65 .4. At present it is not known whether $\theta$ is independent or related to the other bulk exponents $\beta, \nu_{\|}, \nu_{\perp}$.

\section{Finite-size simulations at criticality}

The dynamic exponent $z=\nu_{\|} / \nu_{\perp}$ can easily be verified by finite-size simulations. According to the scaling form (14), several measurements of $n_{0}(t)$ for different system sizes should collapse onto a single curve if $n_{0} t^{\delta_{0}}$ is plotted versus $t / L^{z}$. As shown in Fig. 9, the best data collapse is obtained for $\delta_{0} \simeq 0.27$ and $z \simeq 1.7$, which is in agreement with the known $\mathrm{PC}$ exponents $\delta=0.285(5)$, $z=1.76(3)$, and the previous estimate of $z$ in Sect. IV.

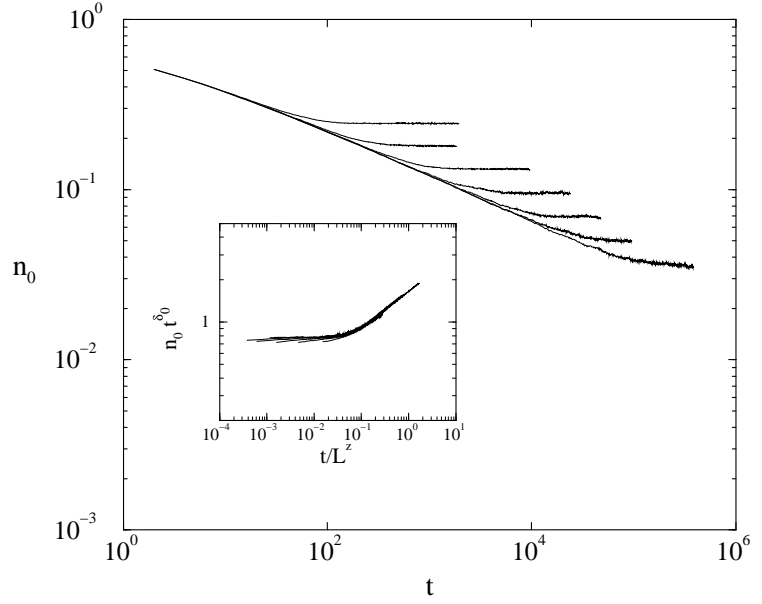

FIG. 9. Finite size results for the parallel model for $L=64,128 \ldots 4096$. The density at the bottom layer $n_{0}(t)$ is plotted as a function of time. The inset shows a data collapse which is explained in the text.

\section{E. Off-critical simulations}

In order to determine the exponent $\beta_{0}$ directly, we perform off-critical simulations in the steady state of the smooth phase. Here we expect the stationary density $n_{0}$ to scale as $\left(p_{c}-p\right)^{\beta_{0}}$, where $\beta_{0} \simeq 0.92(2)$ is the order parameter exponent of the PC class. Surprisingly, a rough estimate of $\beta_{0}$ in standard Monte-Carlo simulations of variants $\mathrm{A}$ and $\mathrm{C}$ yields much smaller values of about 0.6 which deviate from the expected value by more than $30 \%$. Similar deviations are observed if $\nu_{\perp}$ and $\nu_{\|}$are determined in off-critical simulations. For example, the estimate $\nu_{\|}=2.4(5)$ in Sect. IV is much smaller than the expected $\mathrm{PC}$ value $\nu_{\|} \simeq 3.2$.

Off-critical steady state simulations are known to be quite inaccurate because of long transients. Moreover, they are extremely sensitive to errors in the estimation of $p_{c}$. However, in the present case the deviations have a different origin. This can be shown by determining the effective exponent $\beta_{0}(\epsilon)$ which is defined as the local slope of $n_{0}(\epsilon)$ in a log-log representation between the data points $(i-1, i)$

$$
\beta_{0}\left(\epsilon_{i}\right)=\frac{\ln n_{0, i}-\ln n_{0, i-1}}{\ln \epsilon_{i}-\ln \epsilon_{i-1}},
$$

where $\epsilon_{i}=p_{c}-p_{i}$, providing an estimate

$$
\beta_{0}=\lim _{\epsilon \rightarrow 0} \beta_{0}(\epsilon)
$$

As shown in Fig. 10, the effective exponent increases slowly and tends towards the expected PC value as $\epsilon \rightarrow 0$. A simple linear extrapolation yields the estimate 0.9 which is in rough agreement with the expected value of $\beta_{0}$. 

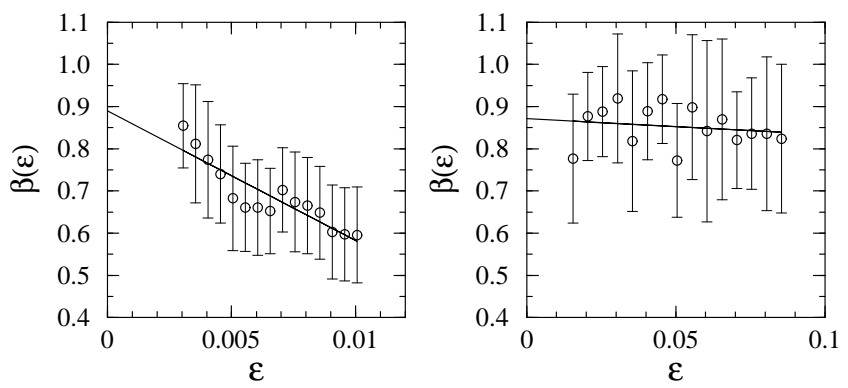

FIG. 10. Left: The effective exponent $\beta_{0}(\epsilon)$ as a function of $\epsilon$ in model $\mathrm{C}$ with $L=24000$ sites. After an equilibration of $4 \times 10^{5}$ time steps the bottom layer density is averaged over $10^{3}$ samples. A simple linear fit gives the estimate $\beta_{0} \approx 0.89$. Right: Corresponding simulation results for the truncated model. Here the effective exponent is almost independent of $\epsilon$. Notice the different ranges of $\epsilon$.

The drift of $\beta_{0}(\epsilon)$ is unusual and may be related to the influence of dynamic processes at higher levels of the interface. To support this hypothesis, we analyze the truncated version of model $\mathrm{C}$ where the heights are restricted to take the values 0 and 1 in order to eliminate the feedback from higher levels. Repeating the same type of analysis at the critical point $p_{c}=0.583(1)$ we observe $\beta_{0}(\epsilon)$ to be in agreement with the expected $\mathrm{PC}$ value over a wide range of $\epsilon$. Thus we are led to the conclusion that dynamic processes at higher levels are responsible for corrections to scaling in the off-critical regime.

\section{F. Unusual scaling for random initial conditions}

So far we have considered the temporal evolution starting from an entirely flat interface. It would be natural to expect the asymptotic scaling behavior to be universal for any initial interface configuration with finite width and short-range correlations. Surprisingly this is not true. For example, starting with random initial conditions $h_{i}=0,1$ the densities $n_{k}$ turn out to decay much slower. For restricted variants we observe an algebraic decay of $n_{0}$ with an exponent $\delta_{0} \simeq 0.13$ which differs significantly from the value 0.275 for flat initial conditions. Similarly, the critical properties of the faceting transition at $p=0.5$ are affected by random initial conditions.

The nonuniversal behavior for random initial conditions is related to an additional parity conservation law. In fact, the dynamic rules (11)-(2) not only conserve parity of the particle number but also conserve parity of the droplet size. Starting with a flat interface the lateral size of droplets is always even, allowing them to evaporate entirely. However, for a random initial configuration, droplets of odd size may be formed which have to recombine in pairs before they can evaporate, slowing down the dynamics of the system.

In the language of BAW2's the additional parity con- servation law is due to the absence of nearest-neighbor diffusion. Particles can only move by a combination of offspring production and annihilation, i.e., by steps of two lattice sites. Therefore, particles at even and odd lattice sites have to be distinguished. Only particles of different parity can annihilate. Starting with a fully occupied lattice all particles have alternating parity throughout the whole temporal evolution, leading to the usual critical behavior at the PC transition. For random initial conditions, however, particles of equal parity cannot annihilate, slowing down the decay of the particle density. BAW2's and other particle processes with parityconserving diffusion will be analyzed in a future study.

\section{G. Comparison with other models}

Recently, Park and Khang proposed a growth model which involves two symmetric particle species [26]. Particles are adsorbed (desorbed) with probability $q(1-q)$ except at those sites where the nearest neighbors are occupied by particles of the other (same) species. Thus, the dynamic rules mimic a PC transition with two symmetric absorbing states [17]. However, the critical exponents at the roughening transition were found to differ significantly from the expected PC values. In fact, once a new layer is completed, the configuration of the previous layer becomes frozen. Thus the kinks between different domains do not act as pinning centers which destroys the $\mathrm{PC}$ transition. Consequently, the interface starts to propagate for $q>q_{c}$.

Another parity-conserving model introduced by Noh et. al. 27] was inspired by interacting monomer-dimer models [16] exhibiting a PC transition. The dynamic rules involve diffusion, order-preserving branching, and order-breaking branching. In absence of order-breaking branching, the interface evolves within a monolayer and undergoes a pre-roughening transition belonging to the PC class. In presence of order-breaking processes several layers may be formed. However, in this case the interface is always rough and the transition is lost.

Even more recently, the same authors investigated a class of parity-conserving growth models 19], generalizing the rules (11)-(2) by the addition of a process for the evaporation of dimers from the middle of plateaus. A similar generalization of monomer models has been studied in the context of nonequilibrium wetting [28]. In both cases the additional evaporation process destroys the stability of the smooth phase. In the dimer models, however, the roughening transition of the dimer models is replaced by a faceting transition between a rough and an (oppositely) faceted phase. Thus, the models of Ref. 19 link anomalous roughening and faceting transitions. 


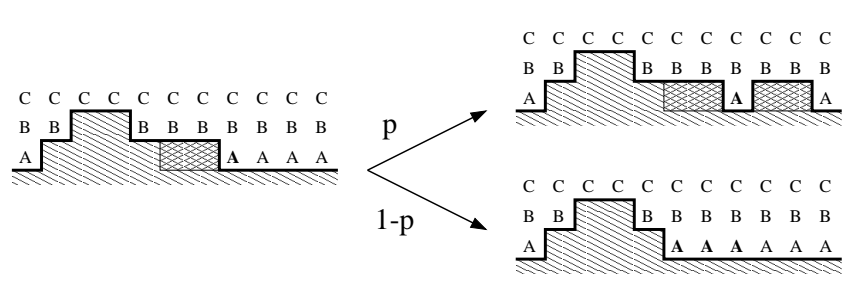

FIG. 11. Extended particle interpretation. Dimers are adsorbed $(2 A \rightarrow \varnothing)$ and desorbed $(A \rightarrow 3 A)$ at the bottom layer. Similar processes take place at higher levels.

\section{UNIDIRECTIONALLY COUPLED BRANCHING-ANNIHILATING RANDOM WALKS WITH EVEN NUMBER OF OFFSPRING}

In order to understand the universal properties of parity-conserving roughening transitions let us extend the particle interpretation. Assuming that $h=0$ is the bottom layer of the interface, we associate particles $A, B, C, \ldots$ with sites at height $h_{i} \leq 0,1,2, \ldots$ (see Fig. 11). As explained before, the dynamic processes at the bottom layer may be interpreted as an effective reaction $A \rightarrow 3 A, 2 A \rightarrow \varnothing$. Similarly, the B-particles on top of the first layer react by $B \rightarrow 3 B, 2 B \rightarrow \emptyset$. Clearly, the temporal evolution of the $B$-particles strongly depends on the actual configuration of the $A$-particles. On the one hand, an $A$-particle implies the presence of a $B$-particle at the same site, giving rise to an effective reaction $A \rightarrow A+B$. On the other hand, both the RSOS constraint and the restriction that dimers react at adjacent sites of equal height may inhibit the above reactions, introducing an effective feedback from higher levels to lower ones. However, as suggested by our numerical results, this type of inhibiting feedback does not affect the critical behavior at the roughening transition.

Similarly, the $C$-particles perform an effective BAW2 on top of the second layer. Therefore, the critical behavior of the first few layers may be described in terms of a simplified particle model where several BAW2 processes are unidirectionally coupled according to the reaction scheme

$$
\begin{array}{lll}
A \rightarrow 3 A & B \rightarrow 3 B & C \rightarrow 3 C \\
2 A \rightarrow \varnothing & 2 B \rightarrow \varnothing & 2 C \rightarrow \varnothing \\
A \rightarrow A+B & B \rightarrow B+C & C \rightarrow C+D, \ldots
\end{array}
$$

generalizing the concept of 'coupled DP' 6]. We propose this reaction scheme to characterize the universal behavior of the dimer models at the roughening transition.

To support this hypothesis, we study the reactions (22) by Monte-Carlo simulations. To this end we use three copies of the BAW2 introduced in Ref. [13] coupled by the rule that a selected $A(B)$-particle instantaneously creates a $B(C)$-particle at the same position, provided that the target site is empty. Using a lattice size of 2500 sites we measure the particle densities $n_{A}, n_{B}, n_{C}$ as functions of time at criticality. As shown in Fig. 12, they display
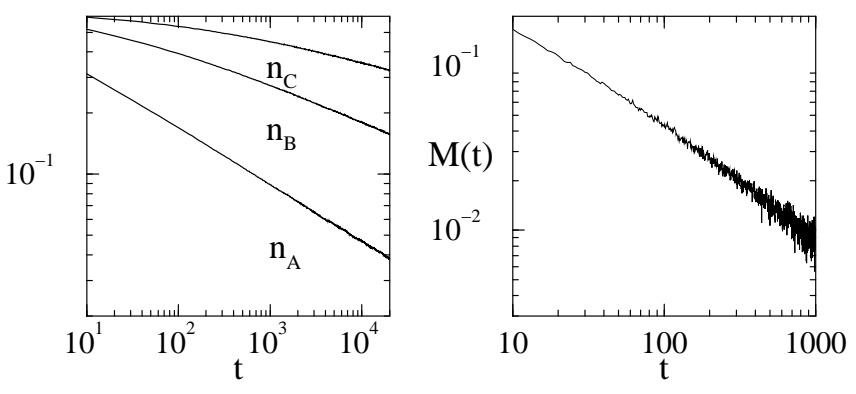

FIG. 12. Unidirectionally coupled PC processes. Left: Densities $n_{A}, n_{B}, n_{C}$ as functions of time. Right: Magnetization $M(t)$ as a function of time averaged over 1000 independent realizations.

essentially the same behavior as the densities $n_{0}, n_{1}, n_{2}$ in the dimer model. Averaging over one decade in time we obtain the exponents

$$
\delta_{A}=0.280(5), \delta_{B}=0.190(7), \delta_{C}=0.120(10),
$$

which are in fair agreement with the corresponding exponents of the dimer model. We also measured the analog of the magnetization $M=n_{A}-n_{B}+n_{C}-n_{D}+\ldots$ in 20 coupled PC processes, obtaining an algebraic decay $|M| \sim t^{-\theta / \nu_{\|}}$with the exponent $\theta / \nu_{\|}=0.74(3)$. This confirms the hypothesis that the roughening transition of the dimer models belongs to the universality class of unidirectionally coupled PC processes. Notice that the coupling $A \rightarrow A+B$ violates parity conservation at the $B$-level. However, using a parity-conserving coupling such as $A \rightarrow A+2 B$ we obtain similar results at the transition.

Before turning to the field-theoretic formulation of coupled PC processes, let us consider the mean field approximation of the reaction scheme (22)

$$
\begin{aligned}
\partial_{t} n_{A} & =\sigma n_{A}-\lambda n_{A}^{2} \\
\partial_{t} n_{B} & =\sigma n_{B}-\lambda n_{B}^{2}+\mu n_{A}, \\
\partial_{t} n_{C} & =\sigma n_{C}-\lambda n_{C}^{2}+\mu n_{B}, \ldots
\end{aligned}
$$

where $n_{A}, n_{B}, n_{C}$ correspond to the densities $n_{0}, n_{1}, n_{2}$ in the growth models. $\sigma$ and $\lambda$ are the rates for offspring production (dimer evaporation) and pair annihilation (dimer deposition), respectively. The coefficient $\mu$ is an effective coupling constant between different particle species. Since these equations are coupled in only one direction, they can be solved by iteration. Obviously, the mean-field critical point is $\sigma_{c}=0$. For small values of $\sigma$ the stationary particle densities in the active state are given by

$$
n_{A}=\frac{\sigma}{\lambda}, n_{B} \simeq \frac{\mu}{\lambda}\left(\frac{\sigma}{\mu}\right)^{1 / 2}, n_{C} \simeq \frac{\mu}{\lambda}\left(\frac{\sigma}{\mu}\right)^{1 / 4}
$$

corresponding to the mean field critical exponents 


$$
\beta_{A}^{M F}=1, \quad \beta_{B}^{M F}=1 / 2, \quad \beta_{C}^{M F}=1 / 4, \ldots .
$$

These exponents should be valid for $d>d_{c}=2$ (see below). Solving the asymptotic temporal behavior we find $\nu_{\|}=1$, implying that $\delta_{k}^{M F}=2^{-k}$. Although this simple mean-field calculation does not include parity conservation, it explains the reduced values of the exponents at higher levels. The different numerical values in one dimension are due to fluctuation corrections which may be computed within a field-theoretic renormalization group approach. A field theory for a single BAW2 was introduced and studied in detail by Cardy and Täuber [14]. Following their notation, the effective (unshifted) action of unidirectionally coupled BAW2's should be given by

$$
\begin{aligned}
& S\left[\psi_{0}, \psi_{1}, \psi_{2}, \ldots, \bar{\psi}_{0}, \bar{\psi}_{1}, \bar{\psi}_{2}, \ldots\right]= \\
& \int d^{d} x d t \sum_{k=0}^{\infty}\left\{\bar{\psi}_{k}\left(\partial_{t}-D \nabla^{2}\right) \psi_{k}-\lambda\left(1-\bar{\psi}_{k}^{2}\right) \psi_{k}^{2}+\right. \\
& \left.\quad+\sigma\left(1-\bar{\psi}_{k}^{2}\right) \bar{\psi}_{k} \psi_{k}+\mu\left(1-\bar{\psi}_{k}\right) \bar{\psi}_{k-1} \psi_{k-1}\right\}
\end{aligned}
$$

where $\psi_{-1}=\bar{\psi}_{-1} \equiv 0$. Here the fields $\psi_{k}$ and $\bar{\psi}_{k}$ represent the configurations of the system at level $k$. However, even for a single copy in $1+1$ dimensions the fieldtheoretic treatment poses considerable difficulties. They stem from the presence of two critical dimensions: $d_{c}=2$, above which mean-field theory applies, and $d_{c}^{\prime} \approx 4 / 3$, where for $d>d_{c}^{\prime}\left(d<d_{c}^{\prime}\right)$ the branching process is relevant (irrelevant) at the annihilation fixed point. Therefore the physically interesting spatial dimension $d=1$ cannot be accessed by a controlled $\epsilon$-expansion down from upper critical dimension $d_{c}=2$. A one-loop calculation for the action (27) would be an even more difficult task.

A fundamental problem of the field theory for coupled DP describing the monomer case is the relevant coupling $\mu$ which grows under renormalization group transformations [6]. Moreover, even in a one-loop calculation certain infrared divergent diagrams are encountered which are proportional to $\mu$. Similar difficulties are expected for unidirectionally coupled PC processes. They might be responsible for violations of scaling in the long time limit appearing as a curvature of $n_{B}$ and $n_{C}$ in Fig. 12 .

\section{PARITY-CONSERVING POLYNUCLEAR GROWTH MODELS}

Roughening transitions related to coupled DP were first observed in so-called polynuclear growth (PNG) processes [7]29] 33]. In these models the interface grows by nucleation processes and deterministic growth of terraces. The use of parallel updates ensures that the maximal propagation velocity is 1 . Depending on the rates for nucleation and terrace growth, the models exhibit a roughening transition from a moving rough phase to a smooth

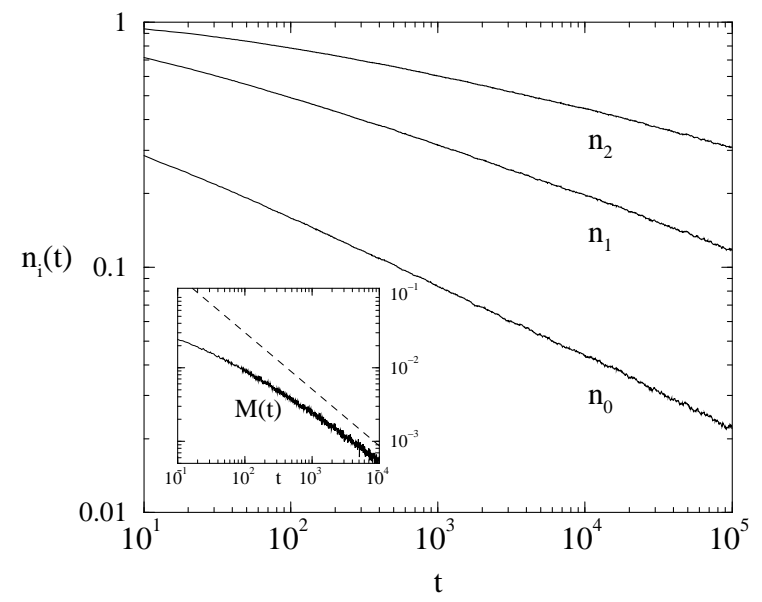

FIG. 13. Parity-conserving PNG model. The densities $n_{0} \ldots n_{3}$ are shown as functions of time. The exponents $\delta_{k}$ are estimated in the interval $10^{3} \leq t \leq 10^{5}$, suggesting that the model belongs to the universality class of unidirectionally coupled PC processes. The inset shows the magnetization $M(t)$. The dashed line indicates the slope -0.77 .

phase propagating at maximal velocity. As shown in Ref. 柾, PNG models may be viewed as upside-down versions of monomer models in a co-moving frame. In some cases it is even possible to relate PNG and monomer models exactly to each other, resolving the apparent paradox that the transition in PNG models requires parallel updates whereas for monomer models the type of updates does not play a role.

In order to understand the DP mechanism, let us consider the PNG model introduced by Kertész and Wolf [7] which is updated synchronously in two sub-steps. At first all up (down) steps of the interface move deterministically to the left (right) over a distance of $u$ lattice spacings. Then all heights are increased with probability $p$. Thus the model is unrestricted. For $u=1$ this dynamic rule can be expressed as a single parallel update

$$
h_{i}(t+1)=\left\{\begin{array}{ll}
m(t)+1 & \text { with prob. } p \\
m(t) & \text { with prob. } 1-p
\end{array},\right.
$$

where $m(t)=\max \left[h_{i-1}(t), h_{i}(t), h_{i+1}(t)\right]$. Starting from a flat interface $h_{i}(0)=0$, the sites at maximal height $h_{i}(t)=t$ may be considered as the active sites of a DP process. The nucleation process turns active into inactive sites with probability $1-p$ while the deterministic growth of terraces resembles offspring production. Therefore, if $p$ is large enough, the interface is smooth and propagates with velocity 1 . Below a critical threshold $p_{c}=0.539(1)$, however, the growth velocity is smaller than 1 and the interface evolves into a rough state. As shown in Ref. [6], this model is a realization of unidirectionally coupled DP processes in a co-moving frame, where the order parameters $n_{k}$ are given by 


$$
n_{k}(t)=\frac{1}{L} \sum_{i} \sum_{h=0}^{k} \delta_{h_{i}(t), t-h} .
$$

In the following we introduce a parity-conserving PNG model which belongs to the universality class of unidirectionally coupled PC processes. The model is defined on a one-dimensional lattice with periodic boundary conditions and evolves by sublattice-parallel updates. In the first half time step pairs of sites $(i, i+1)$ with even $i$ are updated. If $h_{i}(t) \neq h_{i+1}(t)$, the heights are incremented by one step

$$
\begin{aligned}
h_{i}(t+1 / 2) & =h_{i}(t)+1, \\
h_{i+1}(t+1 / 2) & =h_{i+1}(t)+1 .
\end{aligned}
$$

If, however, the two heights are equal, they are updated by the probabilistic rule

$$
\begin{aligned}
h_{i}(t+1 / 2) & =h_{i+1}(t+1 / 2)= \\
& =\left\{\begin{array}{ll}
m(t)+1 & \text { with prob. } p \\
m(t) & \text { with prob. } 1-p
\end{array},\right.
\end{aligned}
$$

where $m(t)=\max \left[h_{i-1}(t), h_{i}(t), h_{i+1}(t), h_{i+2}(t)\right]$. In the second half time step the same update rule is applied to odd pairs of sites. Clearly, this model generalizes the PNG model of Ref. [7] and conserves parity at each height level in a co-moving frame. The conservation law leads to the formation of pinning centers moving at maximal velocity.

Performing Monte-Carlo simulations we observe a roughening transition at the critical threshold $p_{c}=$ $0.5697(3)$. Starting from a flat interface, we measure the densities $n_{k}(t)$ defined in Eq. (29) as functions of time. As shown in Fig. 13, the temporal decay of $n_{k}$ is similar to the one observed in the dimer model. Averaged over two decades in time we obtain the estimates

$$
\delta_{0}=0.28(1), \quad \delta_{1}=0.21(2), \quad \delta_{2}=0.14(2),
$$

which are compatible with the values listed in Table [. The magnetization $M(t)$ defined in Eq. (18) does not show a clean power law behavior but seems to approach an asymptotic decay $t^{-0.77}$ in agreement with previous findings (see inset of Fig. 13). Therefore, we conclude that the roughening transition of the parity-conserving PNG model belongs to the universality class of unidirectionally coupled PC processes.

\section{CONCLUSIONS AND OUTLOOK}

In this paper we have investigated a class of parityconserving growth processes in which dimers adsorb at sites of equal height and desorb at the edges of terraces. At a critical growth rate $p=p_{c}$ the models display a roughening transition from a smooth to a rough phase. In order to demonstrate the robustness of this transition, we have studied four variants of a $1+1$ dimensional parity-conserving growth process with and without RSOS constraint, using either random-sequential or sublattice-parallel dynamics. In addition, we have introduced a parity-conserving polynuclear growth process where a similar transition takes place in a co-moving frame. In all cases the roughening transition is characterized by the same type of critical behavior.

The investigated dimer models generalize previously studied monomer models. Their essential feature is a parity conservation law at each height level, changing the universal properties of the roughening transition. The conservation law leads to the formation of pinning centers separating regions of even and odd parity. Thus, in contrast to the monomer case, the interface remains pinned to the initial height level. At the transition the width is found to increase logarithmically with time due to a slow diffusion and annihilation of the pinning centers. In Eq. (7) we have proposed a finite-size scaling form for logarithmic roughening which is confirmed by high precision simulations. Moreover, we have shown that the universal critical behavior at the first few layers may be described in terms of unidirectionally coupled branching-annihilating random walks with two offspring. Thus we suggest that the concept of undirectionally coupled PC processes defines a whole universality class of parity-conserving roughening transitions.

In case of equal rates for dimer adsorption and desorption all variants undergo a second phase transition where the width increases algebraically. In the restricted case this transition has been identified as a faceting transition from a rough to a faceted phase [19]. In the unrestricted variants, however, we observe a transition from a rough to a freely growing phase characterized by spiky interface configurations.

There are various possible extensions and generalizations of the models studied in this paper. Very recently, Noh et al. 19 investigated a generalization of variant A where dimers may also evaporate from the middle of plateaus. Even at a very small rate this additional process destroys the stability of the smooth phase, turning it into a faceted phase. Remarkably, a sharp transition between the faceted and the rough phase still remains, leading to interesting crossover phenomena between different universality classes which have not been studied so far. It would also be interesting to investigate parityconserving growth processes in higher dimensions. Since the upper critical dimension $d_{c}^{\prime}$ is less than 2 , we expect the roughening transition - if still existing - to be described by mean-field exponents. One may also consider growth processes of $n$-mers where the number of particles at each height level is preserved modulo $n$. Especially in higher dimensions, these $n$-mers might appear in different shapes and orientations. After all it would also be interesting to find experimental realizations for deposition and evaporation of composite particles. 
Acknowledgements:

We would like to thank N. Menyhárd for pointing out the possibility of parity-conserving polynuclear growth processes. H.H. thanks the MTA-MFA in Budapest for hospitality where parts of the work have been done. The simulations were performed partially on the FUJITSU AP1000, AP-3000 and System-V parallel supercomputers. We thank R. Bishop for helping us to operate the System$\mathrm{V}$ machine. G.Ó. gratefully acknowledges support from the Hungarian research fund OTKA (Nos. T025286 and T023552).

[1] H. Hinrichsen and G. Ódor, Phys. Rev. Lett. 82,1205 (1999).

[2] For reviews on growth phenomena and roughening transitions see: J. Krug and H. Spohn, in: Solids far from Equilibrium: Growth, Morphology and Defects, ed. C. Godrèche (Cambridge University Press, Cambridge, 1990); A.L. Barabási and H.E. Stanley, Fractal Concepts in Surface Growth (Cambridge University Press, Cambridge, 1995); J. Krug, Adv. Phys. 46, 139 (1997).

[3] M. Kardar, G. Parisi, and Y.-C. Zhang, Phys. Rev. Lett. 56, 889 (1986); T. Halpin-Healy and Y.-C. Zhang, Phys. Rep. 254, 215 (1995).

[4] U. Alon, M.R. Evans, H. Hinrichsen and D. Mukamel, Phys. Rev. Lett. 76, 2746 (1996); Phys. Rev. E 57, 4997 (1998).

[5] W. Kinzel, in Percolation Structures and Processes, ed. G. Deutscher, R. Zallen, and J. Adler, Ann. Isr. Phys. Soc. 5 (Adam Hilger, Bristol, 1983), p. 425.

[6] U.C. Täuber, M.J. Howard, and H. Hinrichsen, Phys. Rev. Lett. 80, 2165 (1998); Y.Y. Goldschmidt, Phys. Rev. Lett. 81, 2178 (1998); Y.Y. Goldschmidt, H. Hinrichsen, M.J. Howard, and U.C. Täuber, to appear in Phys. Rev. E; H.K. Janssen, cond-mat/9901188.

[7] J. Kertész and D.E. Wolf, Phys. Rev. Lett. 62, 2571 (1989).

[8] J.M. López and H.J. Jensen, Phys. Rev. Lett. 81, 1734 (1998).

[9] G. Bianconi, M.A. Muñoz, A. Gabrielli, and L. Pietronero, cond-mat/9902239.

[10] This class does not include DP-induced passive growth, cf. P. Bhattacharyya, cond-mat/9811160.

[11] H. K. Janssen, Z. Phys. B 42, 151 (1981); P. Grassberger, Z. Phys. B 47, 365 (1982).

[12] H. Takayasu and A. Yu Tretyakov, Phys. Rev. Lett. 68, 3060 (1992); D. ben-Avraham, F. Leyvraz, and S. Redner, Phys. Rev. E 50, 1843 (1994); I. Jensen, Phys. Rev. E 50, 3623 (1994).

[13] D. Zhong and D. ben-Avraham, Phys. Lett. A 209, 333 (1995);

[14] J.L. Cardy and U.C. Täuber, Phys. Rev. Lett. 77, 4780 (1996); J. Stat. Phys. 90, 1 (1998).

[15] N. Menyhárd, J. Phys. A 27, 6139 (1994); N. Menyhárd and G. Ódor, J. Phys. A 29, 7739 (1996).

[16] M.H. Kim and H. Park, Phys. Rev. Lett. 73, 2597 (1994); H. Park, M.H. Kim, and H. Park, Phys. Rev. E 52, 5664 (1995).

[17] H. Hinrichsen, Phys. Rev. E 55, 219 (1997).

[18] G. Ódor, A. Krikelis, G. Vesztergombi and F. Rohrbach: Proceedings of the 7-th Euromicro workshop on parallel and distributed processing, Funchal (Portugal) Feb. 3-5 1999, IEEE Computer society press, Los Alamitos, ed.: B. Werner, ISBN 0-7695-0059-5.

[19] J. D. Noh, H. Park, and M. den Nijs, cond-mat/9812432.

[20] In 11 we introduced this scaling form as $W^{2}(L, t) \simeq$ $\ln \left[t^{a} f\left(t / L^{z}\right)\right]$. Here we use a slightly different notation in order to emphasize that $a$ is an amplitude rather than a critical exponent.

[21] J.R.G. de Mendonca, cond-mat/9903335.

[22] Our earlier conjecture $W \sim \ln t$ in Ref. 1] could not be confirmed by more precise simulations.

[23] F. Family and T. Vicsek, J. Phys. A 18, L75 (1985).

[24] J. M. López, M. A. Rodríguez, and R. Cuerno, Phys. Rev. E 56, 3993 (1997).

[25] P. Grassberger and A. de la Torre, Ann. Phys. (N.Y.) 122, 373 (1979).

[26] S. Park and B. Kahng, preprint cond-mat/9807193.

[27] J. D. Noh, H. Park, and M. den Nijs, cond-mat/9808272.

[28] H. Hinrichsen, R. Livi, D. Mukamel, and A. Politi, Phys. Rev. Lett. 79, 2710 (1997).

[29] D. Richardson, Proc. Camb. Phil. Soc., 74, 515 (1973).

[30] N. Goldenfeld, J. Phys. A 17, 2807 (1984).

[31] J. Krug and H. Spohn, Europhys. Lett. 8, 219 (1989).

[32] C. Lehner, N. Rajewsky, D. E. Wolf and J. Kertész, Physica A 164, 81 (1990).

[33] A. Toom, J. Stat. Phys. 74, 91 (1994); J. Stat. Phys. 74, 111 (1994). 